\title{
Progesterone anti-inflammatory properties in hereditary retinal degeneration
}

\author{
Soledad Benlloch-Navarro ${ }^{\mathrm{a}}$, Laura Trachsel-Moncho ${ }^{\mathrm{a}}$, Ángel Fernández-Carbonella ${ }^{\mathrm{a}}$, Teresa Olivara ${ }^{\mathrm{a}}$, \\ José Miguel Soria ${ }^{\text {a, b}}$, Inmaculada Almansa ${ }^{\text {a, b, },, 1}$, María Miranda ${ }^{\text {a, b, },, 1}$ \\ a Departamento Ciencias Biomédicas, UniversidadCardenal Herrera-CEU Universities, Valencia, Spain \\ ${ }^{\mathrm{b}}$ Instituto de Ciencias Biomédicas, Universidad Cardenal Herrera-CEU Universities, Valencia, Spain
}

\section{A R T I CLE INFO}

\section{Keywords:}

Retinitis pigmentosa

Progesterone

Inflammation

Glia

Malondialdehyde

\begin{abstract}
A B S T R A C T
The interactions between steroid gonadal hormones and the retina (a part of the visual system and the central nervous system (CNS)) have received limited attention and beneficial effects of these hormones in retinal diseases is controversial. Retinitis pigmentosa (RP) is the most common cause of retinal hereditary blindness and to date no treatment is available. However, results regarding the effects of progesterone on the progression of RP are promising. With the idea of demonstrating if the progesterone retinal protection in RP is related to its possible anti-inflammatory properties, we have administered orally progesterone to rd10 mice, an animal model of RP. We observed that progesterone decreased photoreceptors cell death, reactive gliosis and the increase in microglial cells caused by RP. We also examined the expression of neuronal and inducible nitric oxide synthase (nNOS and iNOS), the enzyme responsible for NO production. The results demonstrated a decrease in nNOS expression only in control mice treated with progesterone. Inflammation has been related with an increase in lipid peroxidation. Noticeably progesterone administration was able to diminish retinal malondialdehyde (MDA, a lipid peroxidation product) concentrations in rd10 mice. Altogether, we can conclude that progesterone could be a good therapeutic option not only in RP but also for other retinal diseases that have been associated with inflammation and lipid peroxidation.
\end{abstract}

\section{Introduction}

Neurosteroids refer to those steroids synthesized in the CNS and affecting neurotransmission [1]. In this sense, recent evidences suggest that steroid gonadal hormones play a relevant function not only in the reproductive system but also in others, such as the cardiovascular or the central nervous system (CNS). Neurosteroids include both steroid hormones, which are produced de novo in neurons and glial cells, and their metabolites, which act in the CNS [2,3]. Neurosteroids act in the CNS in an autocrine/paracrine way $[4,5]$, being able to regulate gene expression or affect neurotransmission $[4,6]$. In addition, they possess other biological functions in this system such as the regulation of neuroprotection, neuroplasticity, neurogenesis and neuroinflammation $[7,8]$.

The interactions between gonadal steroid hormones and the retina (a part of the visual system and the CNS) have received limited atten- tion. The eye has long been considered a 'sexually neutral' structure. It was believed there were no differences in physiology and ocular pathology between males and females. Today, we know that the distribution of sex steroid hormones in the eye varies according to sex and age. These differences may explain, in part, the variability in the epidemiology of certain retinal diseases [9].

As with the brain, the retina is a steroid target but also contains a system for its synthesis [10]. Several studies have shown the existence of retinal hormone receptors and steroid enzymes for the formation of ex novo steroids or neurosteroids $[9,11,12]$. In addition, the role of these neurosteroids in the visual function physiology and pathology has been confirmed both in humans and animal models [13,14]. Evidence confirms that there is neurosteroidogenesis in the retina, particularly the de novo production of pregnenolone [10]. The mRNA of the progesterone nuclear receptor has been identified in the retina and in the choroid [15], and progesterone receptor membrane component 1

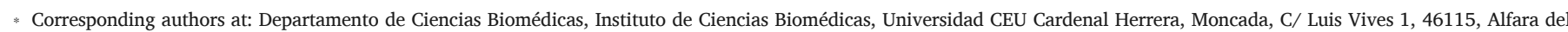
Patriarca, Valencia, Spain.

Email addresses: ialmansa@uchceu.es (I. Almansa); mmiranda@uchceu.es (M. Miranda)

1 Contributed equally.
} 
(PGRMC1) has been detected in photoreceptors and in Müller cells [16].

Nevertheless, there is still controversy about the progesterone advantageous properties in retinal diseases. Some studies in the retina have not succeeded in demonstrating the positive effects of progesterone in a model of ganglion cell damage [17] or in retinal stress induced by light $[18,19]$. Conversely, Lu et al. found that progesterone exerted a protective effect in an intraocular pressure model in rats [20]. There is also evidence suggesting that exposure to estrogens protects against age-related macular degeneration (AMD). These estrogens can be both endogenous (depending on the age at which menarche and menopause occurs and the number of pregnancies) and exogenous (as in hormone replacement therapy and the use of oral contraceptives) [21].

Retinitis pigmentosa (RP) is another retinal disease: the most common cause of hereditary blindness, with a prevalence of one per 4000 individuals [22] and more than one million people affected [23]. The disease is characterized clinically by the progressive loss of rod photoreceptors and, consequently, their function, followed by degeneration of the cones and a decrease in their sensitivity and viability [22,24]. In turn, the main symptoms of this disease are night blindness and degeneration of peripheral vision, which usually appear in adulthood and are due to the already mentioned progressive loss of rods [25]. In addition, an eventual loss of central vision may occur because of macular degeneration of the cones $[25,26]$. Patients with RP may also present vessel attenuation [27], since the loss of vasculature is a metabolic consequence of photoreceptor degeneration [28]. In late stages, patients show an abnormal accumulation of pigment (clusters) in the peripheral retina [26]. Furthermore, the electrical responses to light recorded with electroretinogram (ERG) are abnormal in patients with RP [29]. Symptoms in RP patients generally begin in early adolescence and severe visual dysfunction occurs around 40-50 years of age [30]. However, some patients may experience a rapid progression of the disease over the course of only two decades [24]. On the contrary, others show a slow progression that may never end in blindness [24].

It has been shown that RP causes the activation of an inflammatory response, since retinal antibodies (probably against photoreceptors) have been found in the systemic circulation of patients with RP [31]. Studies that are more recent have highlighted the activation of the macro and microglia preceding the cell death of the photoreceptors. This activation results from multiple biochemical events, including both cytokines and chemokines activation [32].

Results regarding the effects of steroid hormones on the progression of $\mathrm{RP}$ are promising. Recent studies in RP mouse models have shown that the endogenous survival pathways are triggered and cell death is delayed due to norgestrel treatment (a progestin used in hormonal contraceptives) [33]. Our research group has recently demonstrated the protection exerted by progesterone in $\operatorname{rd} 1$ mice, mainly through antioxidant mechanisms [34].

It is known that progesterone acts at multiple levels to interfere with the deleterious cellular processes. Progesterone may decrease oxidative stress, lipid peroxidation, the release of inflammatory cytokines and cellular apoptosis $[35,36]$. The purpose of this study was to demonstrate whether the progesterone retinal protection in RP is related to its possible anti-inflammatory attributes.

\section{Material and methods}

\subsection{Experimental design}

C57BL/6J and rd10 mice were used. Mice were housed in the facilities of the Research Unit of the Department of Biomedical Sciences of the CEU-Cardenal Herrera University. The animals were kept in cages under controlled conditions of temperature $\left(20^{\circ} \mathrm{C}\right)$ and humidity $(60 \%)$ and constant light-dark cycles of $12 \mathrm{~h}$. Animals had free access to water and a standard diet, manufactured and distributed by Harlan Ibérica S.L. (Barcelona, Spain).

Handling and care of the animals was approved by the ethical committee of the CEU-Cardenal Herrera Universities (reference 11/013) and was in accordance with the "Declaration for the use of animals in ophthalmological and vision research" (ARVO; Association for Research in Vision and Ophthalmology).

Day of birth was considered as post-natal day 0 (P0). P15 was chosen as the day to start oral progesterone treatment $(150 \mathrm{mg} / \mathrm{kg}$ weight; progesterone was dissolved in olive oil and administered with the help of a gastric tube). The dose was selected according to a dose-effect curve for progesterone previously published [37]. In order to evaluate progesterone effect, each mouse received oral progesterone administration on alternate days. The las day of treatment was P21. Progesterone has a fast gastrointestinal absorption (serum peak concentrations of three hours) as well as a rapid rate of elimination from the body. Thus, mice were euthanized five hours after the last progesterone dose administration (Fig. 1).

Four experimental groups were used: C57 mice that only received oral administration of the vehicle (olive oil); C57 mice that received progesterone oral treatment; rd10 mice that were administered the vehicle and $\mathrm{rd} 10$ mice that received oral progesterone treatment $(\mathrm{n}=12$ in each group). Our studies were completed on both male and female populations. We have previously demonstrated that progesterone offers similar protection for both sexes and that the effects observed in retina are not gender specific [34].

A group of mice was euthanized at P23 in order to study changes in the evolution of retinal macroglia modifications $(n=4)$.

\subsection{Histological and immunofluorescence studies}

Eyeballs were fixed by immersion in 4\% paraformaldehyde for $2 \mathrm{~h}$, then 3 washes were performed with $0,1 \mathrm{M}$ phosphate buffered saline $\mathrm{pH}$ 7,2 (PBS) for $10 \mathrm{~min}$. Finally, they were cryoprotected using PBS-sucrose in increasing sucrose concentrations $(10 \%-20 \%-30 \%)$ at $4{ }^{\circ} \mathrm{C}$.

Afterwards, $8 \mu \mathrm{m}$ retinal sections were obtained by a Leica CM 1850 UV Ag protect cryostat (Leica Microsistemas SLU, Barcelona, Spain) on superfrost slides (Thermo Fisher Scientific, Braunschweig, Germany) and kept at $-20^{\circ} \mathrm{C}$ until their use.

\subsubsection{Terminal deoxinucleotidyl transferase (TUNEL) assay}

The TUNEL assay was performed with an in situ cell death detection kit (Roche Diagnostics, Mannheim, Germany. To analyse retinal TUNEL positive cells, images were taken with the Nikon DS-Fi1 camera attached to a Leica DM 2000 microscope, with the program Leica appli-

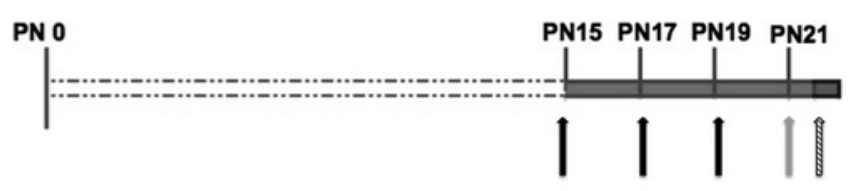

P4 administration

P4 last day administration

Mice sacrifice

Fig. 1. Experimental design. Arrows indicate the days C57 and rd10 mice received progesterone treatment and the post natal (P) day of sacrifice. 
cation Suite version 2.7.0 R1 (Leica Microsystems SLU, Barcelona, Spain).

The TUNEL positive cells were counted manually in the outer nuclear layer (ONL) of three different parts of the retina: central retina, mid peripheral and far peripheral retina. TUNEL positive cells from three retinas were counted for each animal of each group $(n=6)$. The count was taken at $20 \times$ magnification, and the number of cells was referred to the area of the ONL which was used.

\subsubsection{Retinal immunohistochemistry}

Immunofluorescent staining procedures were performed on retinal cryosections that were rehydrated in PBS and merged for $1 \mathrm{~h}$ at room temperature (RT) with blocking solution: $5 \%$ of normal goat serum in PBS-BSA $1 \%$ and Triton $0.3 \%$. Afterwards, they were incubated overnight at $4{ }^{\circ} \mathrm{C}$ with primary antibodies: anti-glial fibrillary acidic protein (anti-GFAP) (1:500, Dako cytomation, Denmark), anti-ionized calcium binding adaptor molecule 1 (anti-Iba1 (1:2000, Abcam, Cambridge, UK), and anti-neuronal nitric oxide synthetase (anti-nNOS) (1:200, Santacruz Biotechnology, Dallas, USA). The next day, sections were washed and incubated for $1 \mathrm{~h}$ in darkness at RT with the fluorescence-conjugated secondary antibody Alexa Fluor 488 (Invitrogen, Life Technologies, Madrid, Spain). Sections were mounted with Vectashield with DAPI (Vector, Burlingame, USA).

Retinal images were taken with a Nikon DS-Fi1 camera attached to a Leica DM 2000 microscope, with the Leica application Suite version 2.7.0 R1 program (Leica Microsistemas SLU, Barcelona, Spain). Representative images were taken of the central, mid peripheral and far peripheral sections of the retina $(20 \times$ magnification).

To evaluate changes in reactive gliosis, the percentage of area occupied by the GFAP antibody labelling was measured throughout the retina $(n=4)$.

To evaluate microglial activation, we counted the total, amoeboid and ramified Iba- 1 positive cells and this value was divided by the ONL area $(n=3)$. We also measured the Iba1- migration index according to the retinal layer where they were located. For this purpose, the method described by Olivares-Gonzáles was used [38].

To evaluate nNOS expression, the number of nNOS positive cells was counted and divided per the selected retinal area $(n=4)$; in this case, it was specifically comprised of the inner plexiform layer to ganglion cell layer. NOS is the enzyme that catalyses the production of NO, a pro-inflammatory substance.

All these measurements were made with the help of the image processing program Image $\mathrm{J}$ 1.45s.

\subsection{Biochemical studies}

\subsubsection{Western blot}

After the mice were euthanized, the eyes were rapidly enucleated and the two retinas of each animal were dissected and homogenized with a $50 \mu \mathrm{L}$ radioimmunoprecipitation (RIPA) buffer ( $n=6$ for the P21 studies and $\mathrm{n}=3$ for the P13 and P18 studies). The homogenization was performed mechanically for $2 \mathrm{~min}$ and kept in ice for $20 \mathrm{~min}$. Subsequently, samples were centrifuged at $13,000 \mathrm{rpm}$ for $10 \mathrm{~min}$ at $4{ }^{\circ} \mathrm{C}$. The supernatant was then removed for protein determination by the Bradford method [39]. Then, $75 \mu \mathrm{g}$ of protein were resolved on $10-15 \%$ acrylamide: bisacrylamide gels. The proteins were moved to membranes of nitrocellulose (GE Healthcare Life Sciences, Barcelona, Spain) and blocked for $1 \mathrm{~h}$ with $0.01 \mathrm{M}$ PBS-Tween $200.1 \%$ with $5 \%$ $\mathrm{w} / \mathrm{v}$ non-fat milk. Membranes were then probed with iNOS antibody (Abcam, Cambridge, UK). Membranes were incubated over night at RT, and bound antibody was detected with a horseradish peroxidase-coupled secondary anti-rabbit (F(ab')2 - HRP, goat anti-rabbit) (Santa Cruz Biotechnology, Santa Cruz, USA). The signal was then recognized with the enhanced chemiluminescence (ECL) developing kit (Amer- sham Biosciences, Buckinghamshire, UK). Finally, blots were quantified by densitometry (ImageQuant ${ }^{\mathrm{TM}} \mathrm{TL}, \mathrm{GE}$ Healthcare Life Sciences, Barcelona, Spain).

\subsubsection{Malondialdehyde concentration determination}

Similar to the process above, the eyes were enucleated directly after the mice were euthanized, and the two retinas of each animal $(n=6)$ were dissected and homogenized at 13,000 rpm for $1 \mathrm{~min}$ with an ultraturrax T25 (Labortech, Staufen, Germany) in phosphate buffer $0.1 \mathrm{M}$ at $\mathrm{pH}$ 7.2. Retinal homogenates were centrifuged at $6000 \mathrm{rpm}$ for $2 \mathrm{~min}$ at $4{ }^{\circ} \mathrm{C}$. The supernatant was used for the determination of proteins by Lowry's method [40] and for the determination of malondialdehyde (MDA).

In order to quantify MDA concentration, a modification of the method presented by Richard [41] was used.

\subsubsection{Induction of lipid peroxidation in mice tissue homogenate}

Liver tissue from six C57BL/6J mice was used, and, $10 \mathrm{ml}$ of $0.1 \mathrm{M}$ PB was added to a sterile $50 \mathrm{ml}$ (Falcon) container containing the two livers. The homogenate was obtained with the help of an ultraturrax at $13,000 \mathrm{rpm}$ for $5 \mathrm{~min}$. Then, the homogenate was centrifuged at $6000 \mathrm{rpm}$ for $5 \mathrm{~min}$. Finally, the remaining supernatant was frozen and aliquoted at $-20^{\circ} \mathrm{C}$ until use.

Lipid peroxidation was induced by adding salts of $\mathrm{Fe}$ (II) and ascorbic acid to homogenate tissues and $0,0.5,1,2,4$ and $10 \mu \mathrm{M}$ of progesterone were also added. The modified experimental model of Fraga et al. [42] was used.

\subsection{Statistical analysis}

The results are presented as mean values \pm standard deviation. Variance homogeneity was determined by Levene's Test of Variance Homogeneity. The analysis of variance (ANOVA) was used. When the ANOVA indicated a significant difference, the Fisher's LSD or the Games-Howell was performed. SPSS software package version 15.0 was used.

\section{Results}

\subsection{Progesterone decreases photoreceptor cell death in the rd10 retina}

Fig. 2A shows the TUNEL cell labelling in the far peripheral retina of the mice belonging to each group used in this work (C57, progesterone treated C57, rd10 and progesterone treated rd10 mice). RP animal models, including the rd10 mouse model, show a severe photoreceptor cell death that can be easily demonstrated with the help of the TUNEL assay. The results obtained in the present study show an increase in TUNEL positive cells in the untreated rd10 mice (Fig. 2B) because of the degeneration.

Herein, the progesterone effect on retinal cell death and degeneration has been studied in three different areas (far peripheral, mid peripheral and central retina) of the control and rd10 mice retina (Fig. $2 \mathrm{~B})$. We observed that the number of TUNEL positive cells is increased in the untreated rd10 mice in the far peripheral retina when compared to all other animal groups (* $\mathrm{p}<0.007 v$ s. all groups). However, in this retinal area, the progesterone treated rd10 mice showed a significant decrease in the number of retinal dying cells compared to control retinas. A statistically significant increase in TUNEL positive cells in the rd10 retina was also observed compared to control mice in both mid peripheral and central retina (\# $\mathrm{p}<0.005$ vs. C57 groups). Interestingly, TUNEL labelled cells decreased in these two retinal areas when the animal received progesterone oral treatment, though statistically significant differences were not found (Fig. 2B). This different response to progesterone treatment of the different retinal areas studied may be 
A
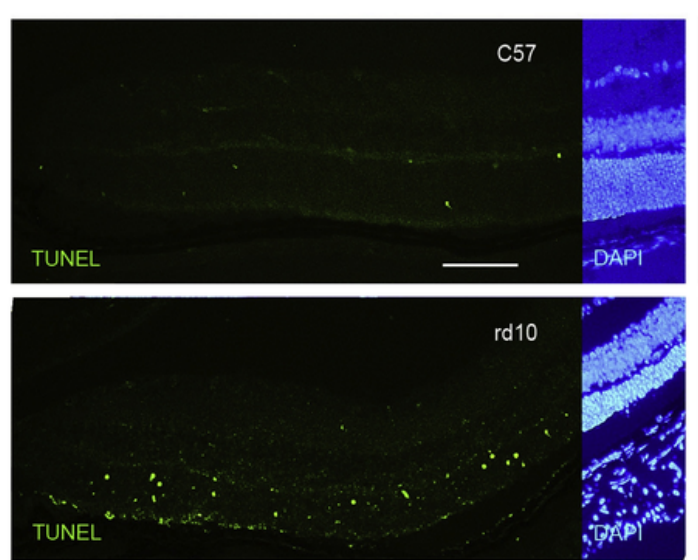

B 5000

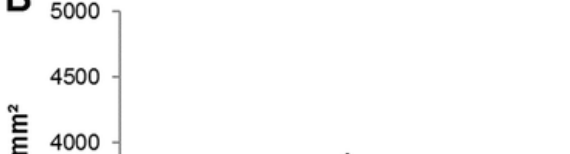

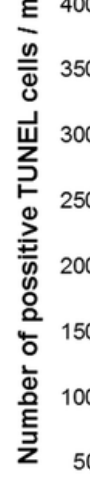

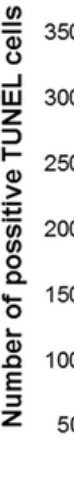

C

\begin{tabular}{|c|c|c|c|c|c|c|c|c|c|}
\hline & \multicolumn{2}{|c|}{ Far peripheral retina } & \multirow{2}{*}{$\begin{array}{l}\% \text { of TUNEL reduction } \\
\text { rd10 treated vs rd10 }\end{array}$} & \multicolumn{2}{|c|}{ Mid peripheral retina } & \multirow{2}{*}{$\begin{array}{l}\% \text { of TUNEL reduction } \\
\text { rd10 treated vs rd10 }\end{array}$} & \multicolumn{2}{|c|}{ Central retina } & \multirow{2}{*}{$\begin{array}{l}\% \text { of TUNEL reduction } \\
\text { rd10 treated vs rd10 }\end{array}$} \\
\hline & rd10 & rd10 treated & & rd10 & rd10 treated & & rd10 & rd10 treated & \\
\hline Average of TUNEL cells/mm² & 2633,55 & 912,43 & \multirow{2}{*}{65,35} & 2844,85 & 1408,49 & \multirow{2}{*}{50,49} & 3304,44 & 1832,34 & \multirow{2}{*}{44,55} \\
\hline Standard deviation of the mea & 893,10 & 351,61 & & 896,60 & 856,06 & & 1420,27 & 1790,27 & \\
\hline
\end{tabular}

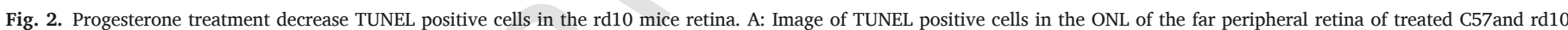

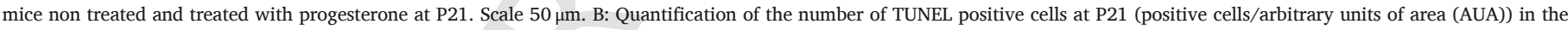

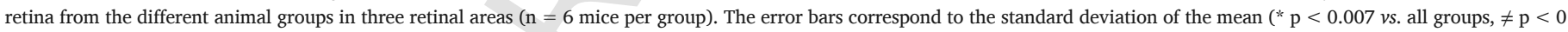
.04 vs. C57 treated; \# p < 0.005 vs. C57 groups). C: Mean values, standard errors and percentage of improvement between non treated and treated with progesterone rd10 mice.

related to the fact that there is a gradient in the rd10 degeneration, from the centre to the periphery of the retina.

Fig. 2C shows the percentage of decrease in the number of TUNEL cells in the rd10 mice in the different areas of the retina. We observed that the greatest decrease in TUNEL positive cells takes place in the far peripheral retina, with more than $65 \%$ of reduction. However, in the mid peripheral and in the central retina, a decrease in the number of TUNEL labelled cells of approximately $50 \%$ and $40 \%$ was also detected.

\subsection{Progesterone induces modifications in the rd10 mice retinal gliosis}

An increase in gliosis and in the reactivity of glial fibrillary acidic protein (GFAP) in the retina takes place during the degeneration process in the rd10 mice [43]. In order to evaluate the possible effect of progesterone on this characteristic reactive gliosis, the labelling of the GFAP protein in the different retinal areas has also been studied at P21 and P23.
Fig. 3A shows the quantification of the occupied area of the retina by the GFAP staining at P21 for the different experimental mice groups (C57, progesterone, treated $\mathrm{C} 57, \mathrm{rd} 10$ and progesterone treated $\mathrm{rd} 10$ mice) in each of the areas of the retina. We found statistically significant differences in these values in all areas of the retina among all groups of animals (*p $<0.02 v s$. all groups). Interestingly, we observed the characteristic gliosis typical of the rd10 retina, but we also observed that progesterone treatment increased GFAP reactivity in both control (C57) and rd10 mice.

As previously stated, the study of the GFAP protein was carried out on P23 (Fig. 3B), with the purpose of determining whether the induced progesterone reactivity was chronic or if it could be an acute effect that could revert with time. Our results show that at P23, there is an increase in the labelling of GFAP in the rd10 retinas compared to the control retinas. However, at P23 progesterone treatment significantly decreases this gliosis in the rd10 animals. This decrease is statistically significant in the far peripheral retina. 

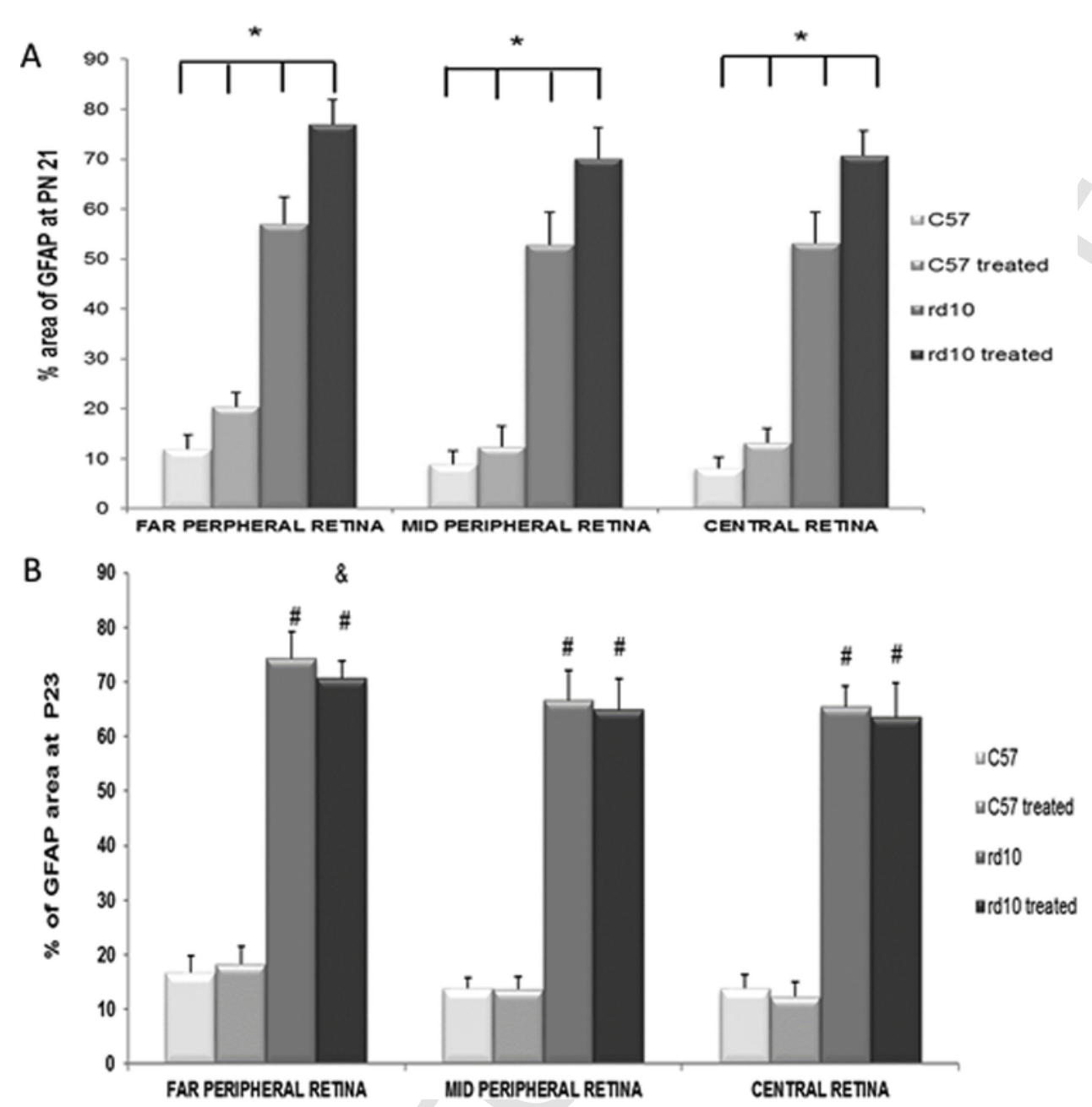

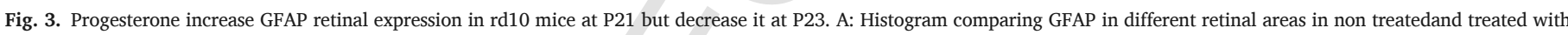

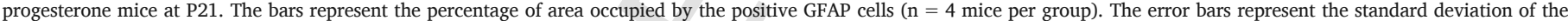

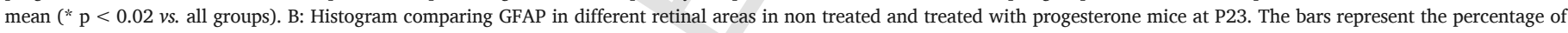
area occupied by the positive GFAP cells ( $\mathrm{n}=4$ mice per group). The error bars are the standard deviation of the mean ( \& $\mathrm{p}<0.005 v s$. rd10; \# $\mathrm{p}<0.01$ vs. C57 groups).

Fig. 4A shows an example of the GFAP staining of untreated and progesterone treated retinas at P21 and P23. We also determined the percentage of increase or decrease in GFAP retinal staining at P23, with regard to the staining observed at P21 (Fig. 4B). In the central retina, the typical gliosis of this degeneration increased approximately $12 \%$ at P23 in rd10 mice when compared to P21. However, in the rd10 group treated with progesterone, there is a decrease in the gliosis at P23, with more than $7 \%$ of reduction in GFAP labelling.

\subsection{Micoglia alterations in the rd10 retina and progesterone effect}

We studied whether progesterone treatment was able to reduce microglial activation in rd10 retinas. Iba1 (microglia/macrophage-specific calcium-binding protein) was used to identify microglial cells. We also determined the migration level from the inner to the outer layers of retina as indicated by Olivares-González et al. [38].

Iba1 immunolabeling was upregulated in rd10 retinas compared to control retinas (Fig. 5A). Progesterone significantly reduced Iba1-positive immunolabelling and migration to outer layers compared to non-treated rd10 retinas (Fig. 5B and C).

\subsection{Progesterone effects on the neuronal nitric oxide synthase (nNOS)}

Part of the progesterone neuroprotective actions may also involve the reduction of pro-inflammatory substances, such as nitric oxide (NO) [44]. nNOS is the most predominant isoform of NOS in the normal retina [45]. In order to evaluate the possible effect of progesterone on the expression of those enzymes related with NO synthesis, we performed immunohistochemistry for nNOS in the P21 retinas of control, rd10 and progesterone treated mice.

Fig. 6A shows images of the nNOS immunohistochemistry in different control and rd10 mice retinal areas. nNOS labelling can be observed in the ganglion cell layer (CCG), the inner plexiform layer (IPL) and the amacrine cells of the inner nuclear layer (INL). Data on nNOS immunostaining in the four animal groups already mentioned are showed in Fig. 6B. In control mice, progesterone produced a significant decrease in the total number of nNOS-expressing cells compared to the rest of the groups being studied (* $\mathrm{p}<0.02 v$ s. all groups). In addition, in the far peripheral retina, an increase in nNOS protein was observed in the progesterone treated rd10 mice when compared to the untreated C57 group (\#p $<0.0001$ vs. C57). 
A

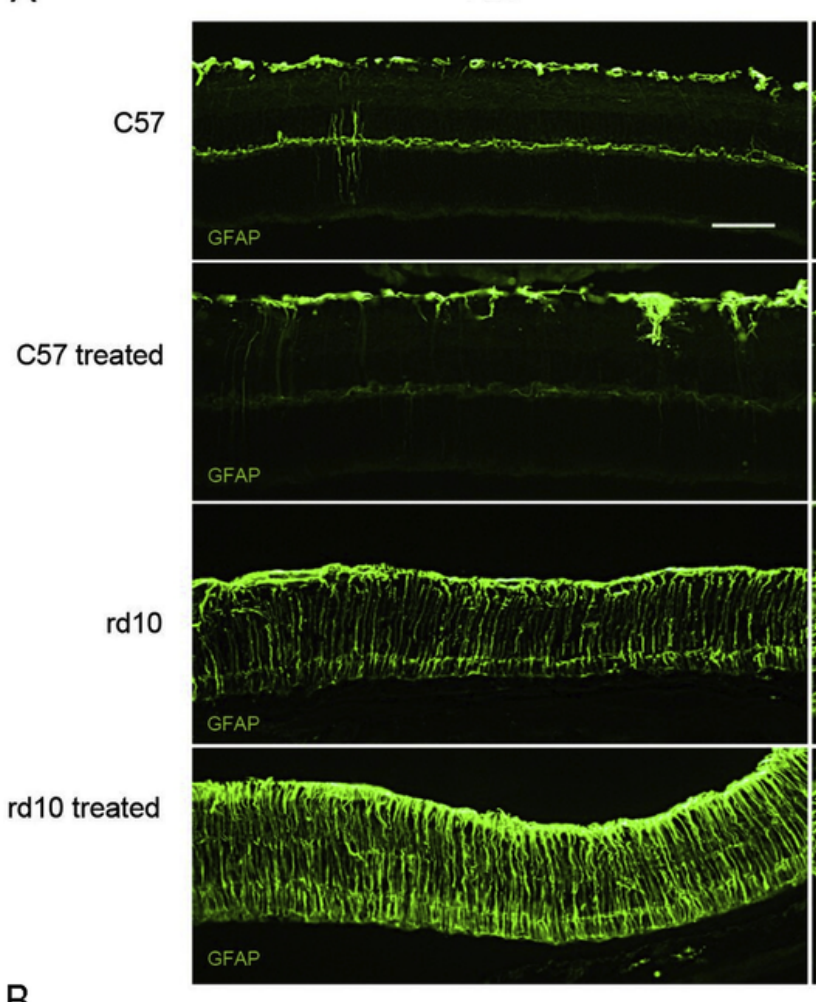

P23

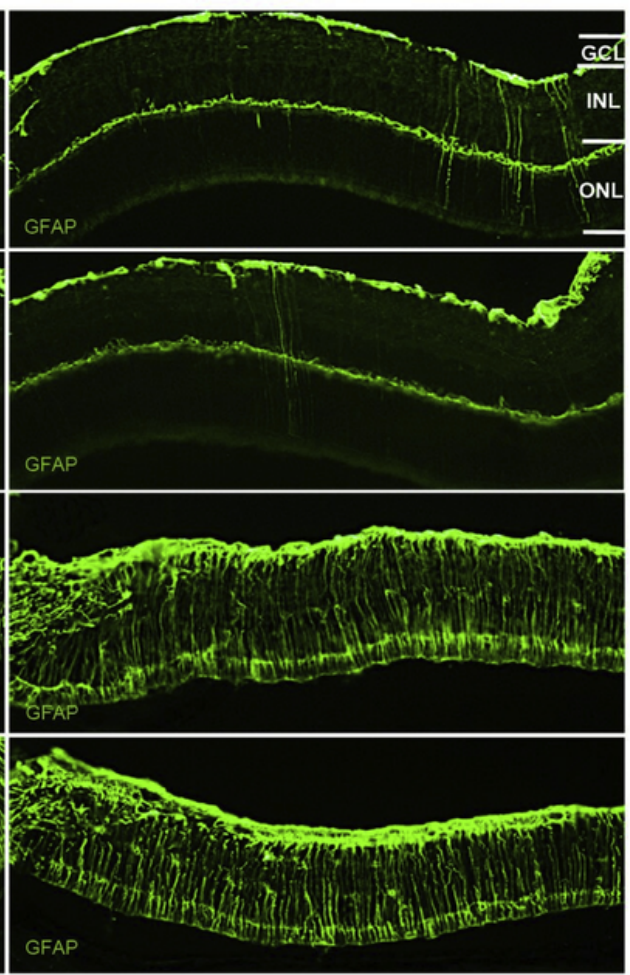

B

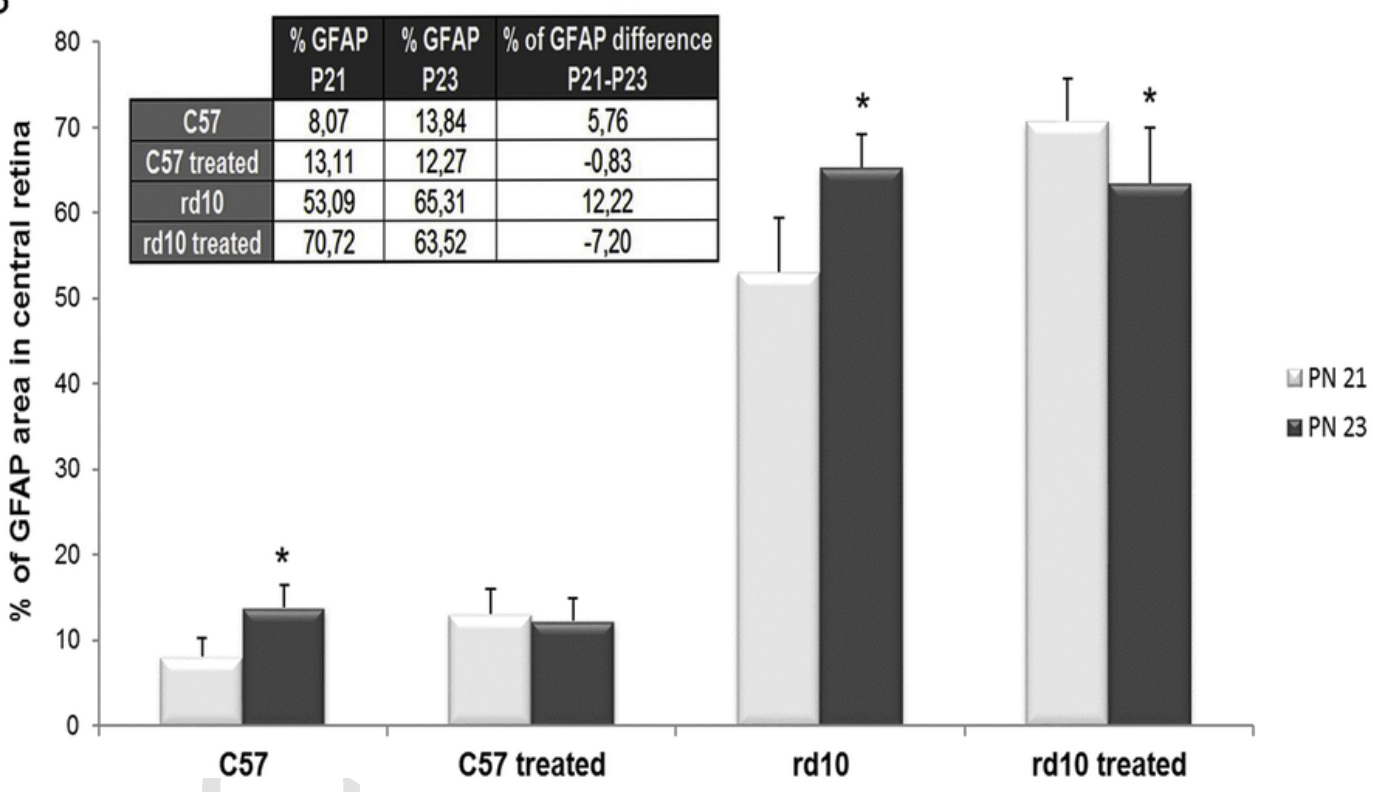

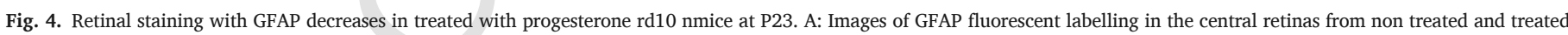

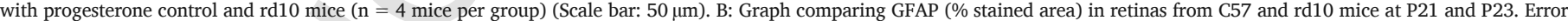

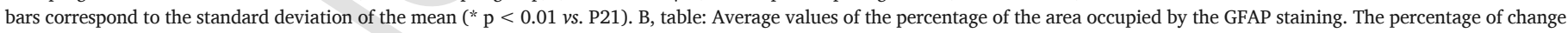
(increase or decrease of staining) in the GFAP area between days P21 and P23 is also shown.

\subsection{Alterations in inducible nitric oxide synthase in rd10 retina (iNOS)}

Fig. 7A and B show an example of the western blot assay for iNOS expression and the quantification of the iNOS protein at P21 in the retina of both untreated C57 and r10 mice and those treated with progesterone. There is a statistically significant decrease of the optical density values of iNOS at day P21 in the untreated and treated rd10 mice versus control mice (* $\mathrm{p}<0.04$ vs. C57; $\neq \mathrm{p}<0.01$ vs. C57 treated).
The presence of iNOS has been demonstrated in the outer segment of photoreceptors in the retina of other mice models of retinal degeneration, including the decrease of its expression with the photoreceptor cell death. [46]. To demonstrate if a similar loss of iNOS expression is also due to the loss of photoreceptors with time in the rd10 mice, this expression was also studied at P13 and P18 (Fig. 8A and B). A significant decrease in iNOS was demonstrated in rd10 retina at P18 (when the loss of photoreceptors is evident in rd10 mice) compared with P13 (\& $\mathrm{p}<0.03$ vs. P13 rd10). 

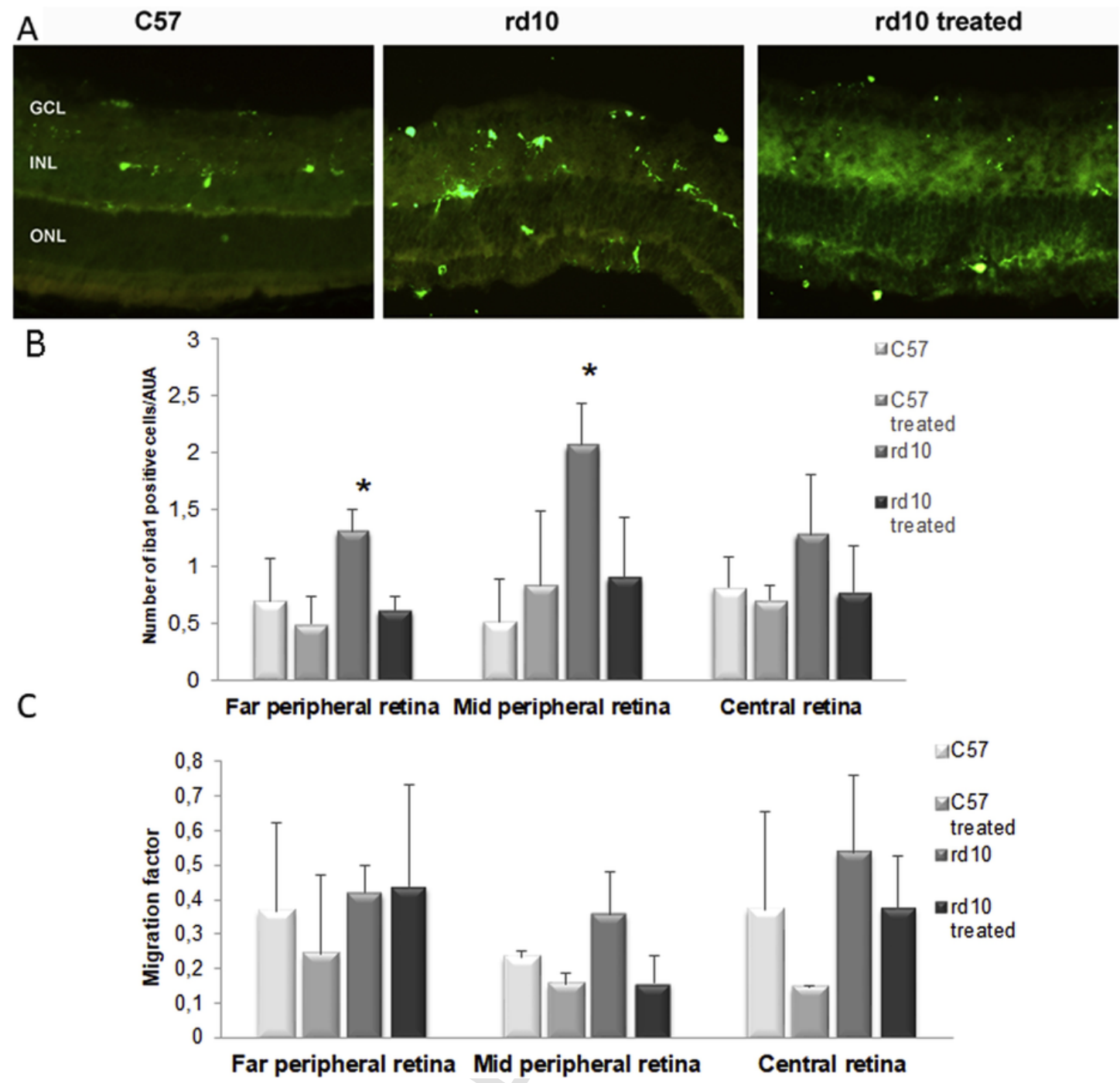

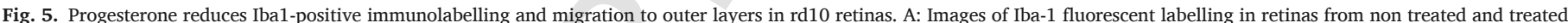

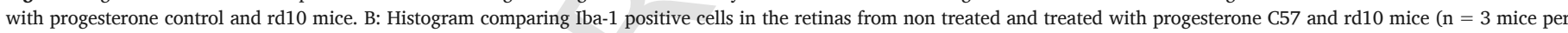

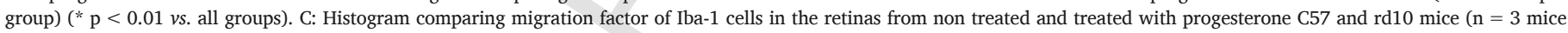
per group) (* $\mathrm{p}<0.01$ vs. treated C57 and rd10 groups; \# $\mathrm{p}<0.01$ vs. treated C57 group).

\subsection{Progesterone-induced effects on lipid peroxidation}

The retina concentrations of malondialdehyde (MDA) have been determined at P21 in the different study mice groups (untreated C57 and rd10 mice and those treated with progesterone) in order to establish the possible role of progesterone on lipid peroxidation. Fig. 9A shows that there is a significant increase in the MDA concentration in the retina of the untreated rd10 mice compared to the C57 group (\#p $<0.006$ vs. C57). In addition, treatment with progesterone in the rd10 mice is capable of decreasing MDA concentration to similar values of those detected in C57 mice. This decrease in MDA concentration induced by progesterone is also observed in C57 treated mice $(* \mathrm{p}<0.001 v$ s. untreated C57).

To further evaluate this progesterone antioxidant property, we have studied the progesterone action in an ex vivo and chemical lipid peroxidation induction experiment. To this end, lipid peroxidation induction in liver tissue has been carried out with Fe (II) iron salts and ascorbic acid. Liver tissue was obtained from the same six C57 animals used in this study, and liver was used instead of retina because of the amount of tissue needed for this experiment. In the present work, it has been also demonstrated that progesterone possesses the ability to inhibit ex vivo lipid peroxidation in a dose-dependent manner. Fig. 9B shows that there is a negative and significant correlation $\left(R^{2}=0.9572 ; p<0.01\right)$ between the use of increasing concentrations of progesterone and the concentration of MDA in mice liver.

\section{Discussion}

RP is a genetically complex disease [47]. Therefore, therapeutic strategies are mainly aimed at inhibiting apoptosis, promoting neuroprotection and repairing retinal pigment epithelium (RPE) and photoreceptor cells.

Neuroinflammation is considered a hallmark of many chronic degenerative disorders [48]. As in other retinal pathologies, inflammation plays a fundamental role in RP [49]. Taking this fact into consideration, the inhibition of inflammation could be a good option to promote or increase photoreceptor protection.

Several studies demonstrate the anti-inflammatory role of progesterone in the brain [50-53]. It has also been shown that progesterone can reduce inflammation in tissues other than the CNS, such as the intestine [54]. In this study, we demonstrate that the beneficial effect of 
A
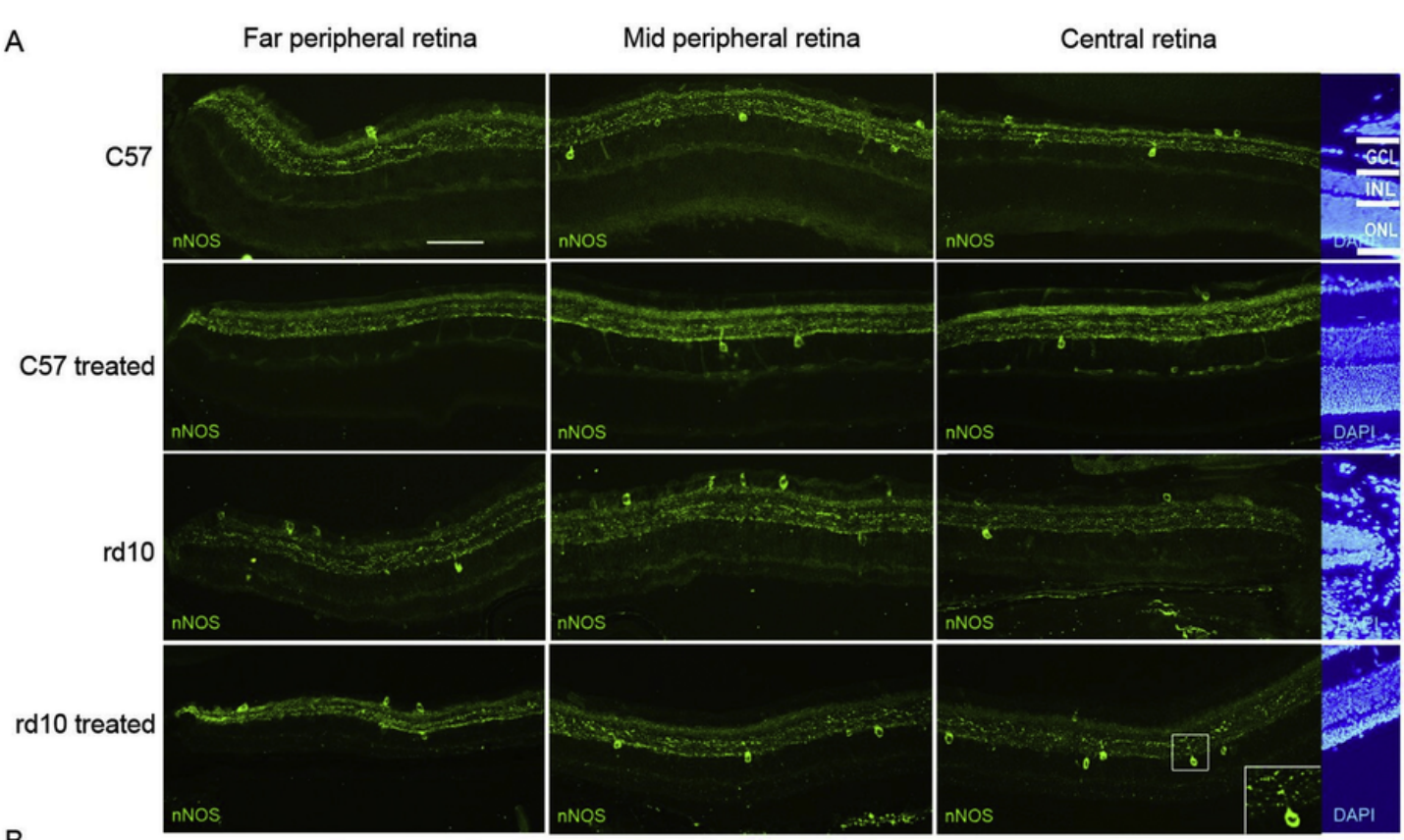

B
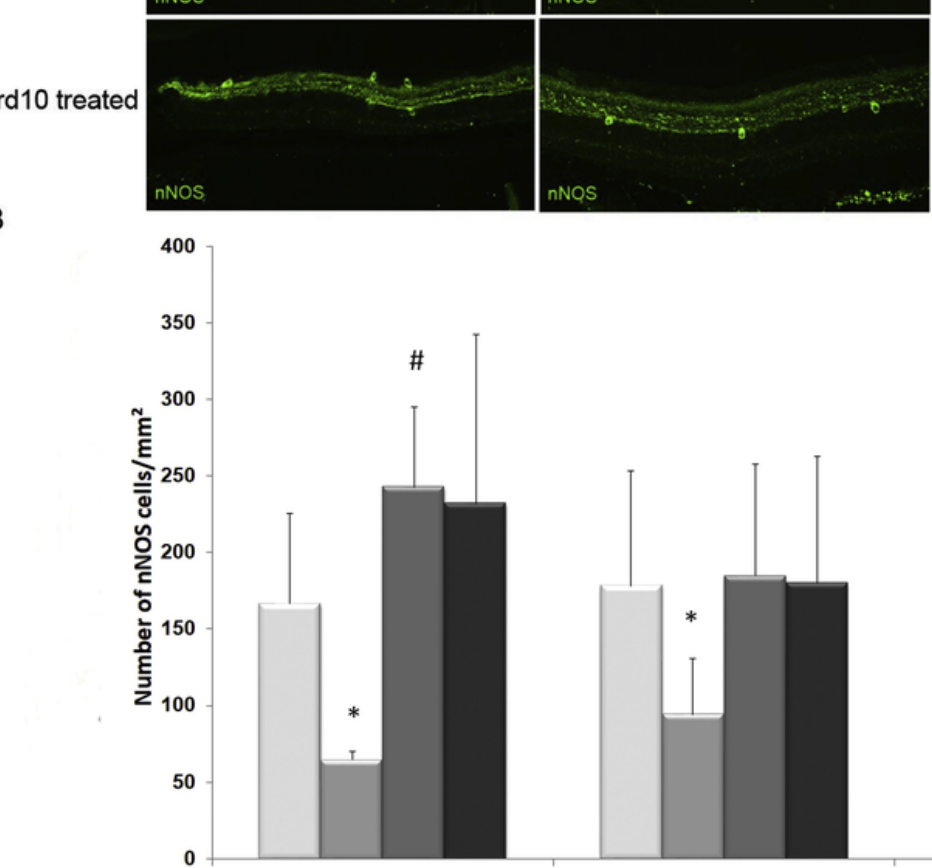

Far peripheral retina

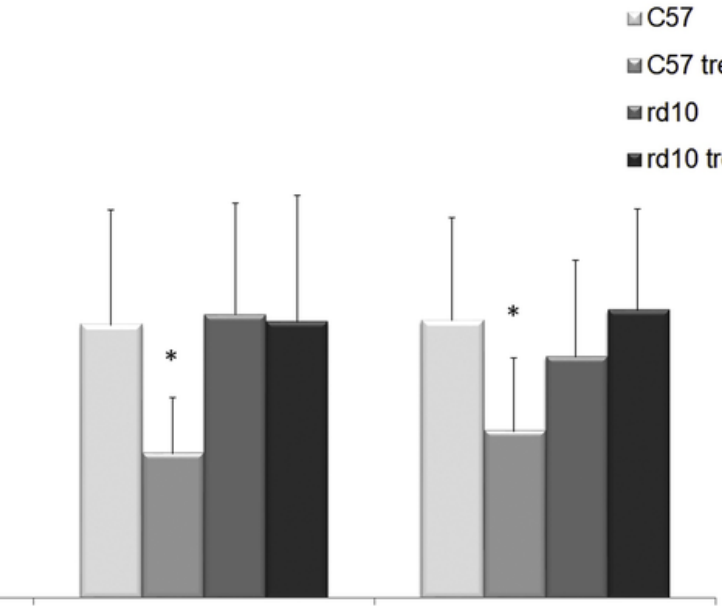

$\mathrm{C} 57$

ard10

rd10 treated

Mid peripheral retina

Central retina

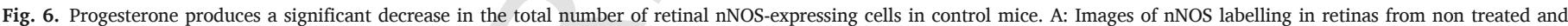

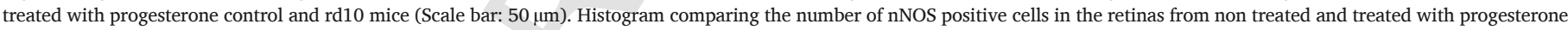
C57 and rd10 mice ( $\mathrm{n}=4$ mice per group) $\left({ }^{*} \mathrm{p}<0.02\right.$ vs. all groups; $\# \mathrm{p}<0.0001$ vs. C57).

progesterone in the RP retina could be related to its potential retinal anti-inflammatory properties.

\subsection{Progesterone protects photoreceptor cells in the rd10 retina}

Herein, we demonstrate that in rd10 mice, progesterone significantly decreases TUNEL positive photoreceptors in the far peripheral retina (Fig. 2). In the rd10 mice, rods begin to degenerate between day P16 and P20, with a maximum of cell death between P20 and P25 occurring in a gradient from the centre to the periphery, so that the degeneration in the periphery is delayed by $2-3$ days $[55,56]$. This may explain the greater effect shown by progesterone in the far peripheral retina. Our results also confirm previous studies concerning the effect of progestin on the delay of cell death in rd10 mice [33]. Previously, we have also demonstrated that progesterone was able to decrease photoreceptor death and to improve retinal function (electroretinogram) in a different RP animal model, the rd1 mice [34].

\subsection{Progesterone effect on retinal macro and microglial cells}

Müller cells are the predominant macroglial cells in the retina. An increase of GFAP staining is observed in macroglial cells in all retinal diseases, including glaucoma, AMD, retinal detachment and RP [57]. In control retinas, the expression of GFAP is localized in astrocytes. However, in several RP models, GFAP is also expressed in Müller cells during the degenerative process [56]. The upregulation of GFAP can be used as a sensor of retinal injury and Müller cells activation [58].

There are different gliosis states that range from mild to moderate. In the mild state, glial cells can be subjected to hypertrophy and changes in functionality, although axonal regeneration can be favoured. An acute activation of the glia is neuroprotective, but continuous activation is harmful because the cells that are under hypertrophy lose their function and form glial scars that inhibit axonal regeneration and neuronal survival [49]. 


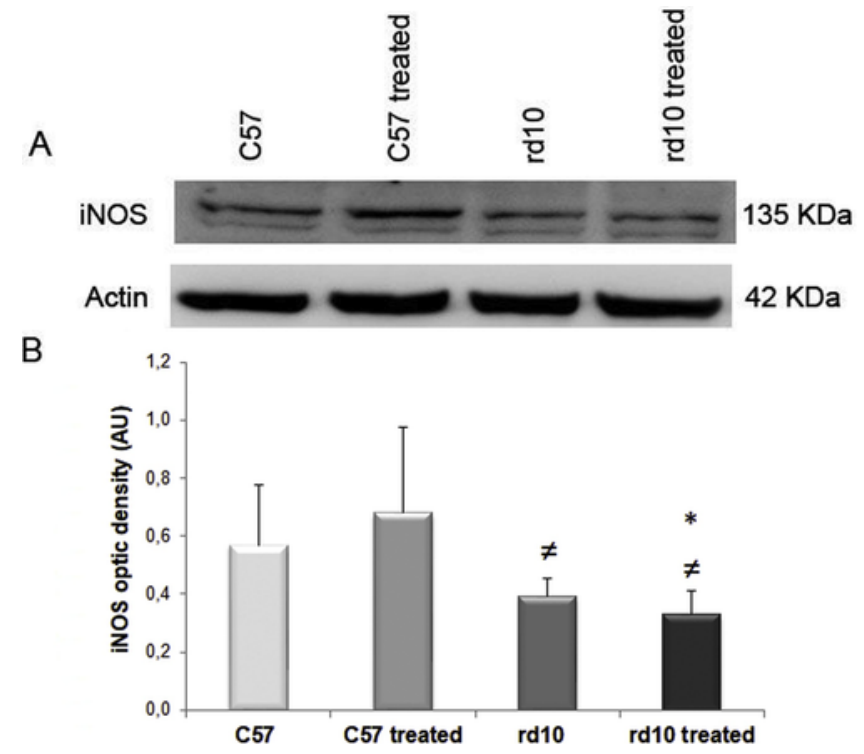

Fig. 7. iNOS expression is decreased in rd10 retinas compared to control retinas at P21. A: Western-blot bands images for control and rd10 mice ( $n=6$ per group). (B) Histogram represents the bands optical density quantification (iNOS/Actin ratio) in each group. The error bars represent the standard deviation of the mean (* $\mathrm{p}<0.04$ vs. C57; $\neq \mathrm{p}<0.01$ vs. C57 treated).

A

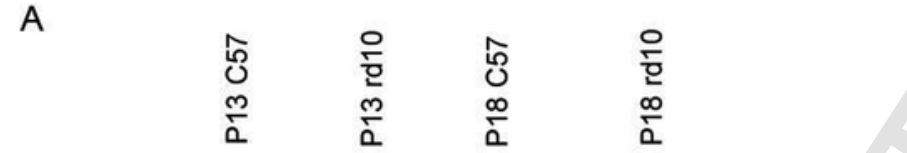

$135 \mathrm{KDa}$

$42 \mathrm{KDa}$

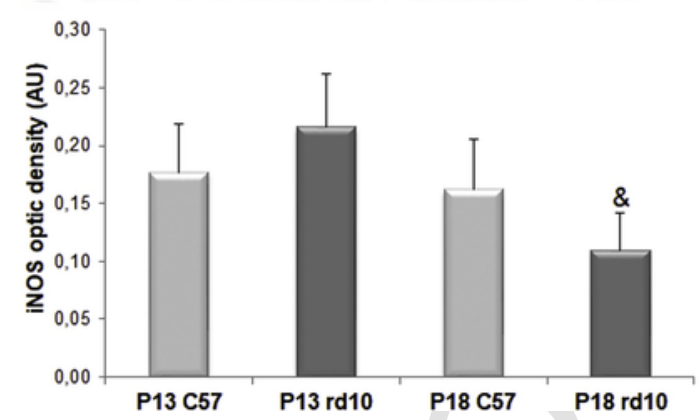

Fig. 8. iNOS expression is decreased in rd10 retinas at P18 compared with P13. A: Image of the western-blot bands for both the control and rd10 mice ( $\mathrm{n}=3$ per group). (B) The graph represents the optical density quantification of the bands (iNOS/Actin ratio) for each experimental group. The error bars represent the standard deviation of the mean (\& $\mathrm{p}<0.03$ vs. P13 rd10).

Our results confirm that there is a reactive gliosis associated with degeneration in the rd10 mice. Moreover, the treatment with progesterone can cause an increase of rd10 and C57 Müller cells reactivity at P21 (Fig. 3). However, at P23 progesterone treatment significantly decreases the GFAP labelling in the rd10 animals (Fig. 4). These results may suggest that progesterone induced first an acute glial activation and that was later able to decrease the harmful chronic glial proliferation, thus avoiding the glial scar. Anti-inflammatory progesterone properties have also been demonstrated in other tissues such as hippocampus [59]. It has been suggested that progesterone effects on reactive gliosis may be mediated by some of its metabolites, such as allopregnanolone (ALLO), and that progesterone inhibition metabolism to allo-
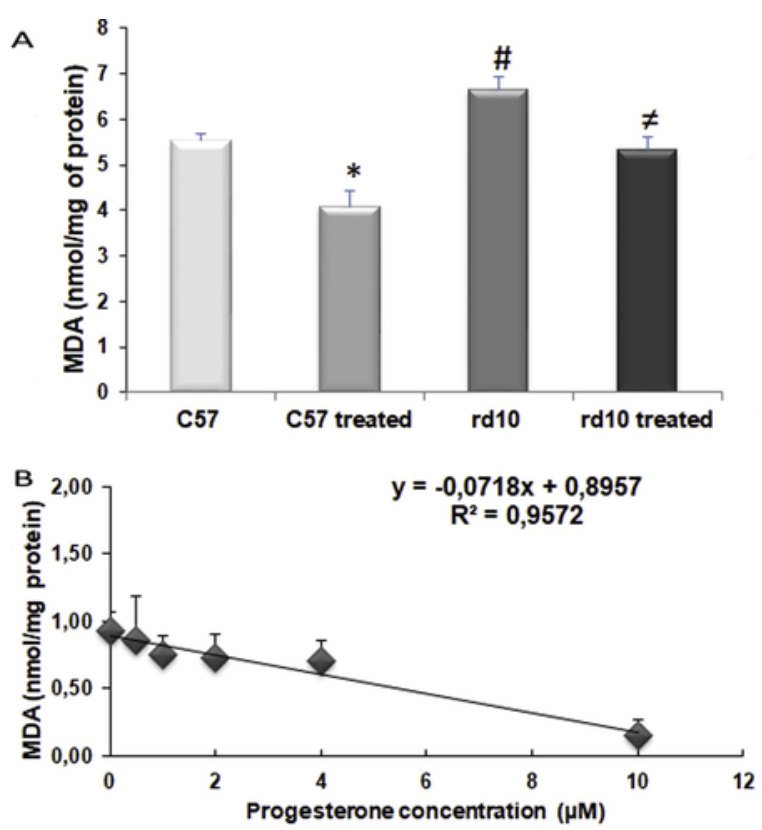

Fig. 9. Progesterone decreases lipid peroxidation. A: Concentrations of MDA (nmol/mg protein) in retinas of not treated and treated with progesterone C57 and rd10 mice at P21. The bars represent the mean ( $n=6$ animals for group) of the MDA concentrations for the different study groups. The error of the bars are the standard deviation of the mean (* $\mathrm{p}<0.001$ vs. C57 and untreated $\operatorname{rd10}$; $\mathrm{p}<0.006$ vs. C57; $\neq \mathrm{p}<0.003$ vs. C57 treated and rd10). B: Effect of the application of different progesterone concentrations (0 - 0, 5 $1-2$ - 4 and $10 \mu \mathrm{M}$ ) on the concentration of MDA in a tissue homogenate (nmol / mg protein) determined by HPLC. The error bars represent the standard deviation of the mean.

pregnanolone blocks this antigliotic action [60]. Other studies also consider the therapeutic potential of ALLO as it has been shown that it has a role in modulation of plasticity in certain eye and brain disorders [61].

A third type of glial cell in the retina is microglia, the resident immune cell. The physiological role of microglia in the retina has not yet been completely clarified, but in chronic inflammation, microgia cell activation is deleterious and involves the release of proinflammatory factors and increases in oxidative and/or nitrosative stress [62]. Additionally, in response to an injury, activated microglial cells can migrate to the affected site [62].

In our study, we assessed microglial activation using the classical microglia marker Iba1, and we determined a migration factor (as explained in the material and methods section). Our results show that the number of Iba1-positive cells were significantly increased in the far and mid peripheral retina of rd10 mice when compared to the control group. Additionally, a significant decrease of more than 30\% was observed when these animals were treated with progesterone (Fig. 5A and B). In the rd10 retinas, the microglial migration factor was significantly higher than in control retinas, indicating that cells are migrating to the retinal outer layers. After progesterone treatment, we observed a lower microglial migration factor in the mid peripheral and central retina, which suggests that these cells were located mainly in the retinal inner layers (Fig. 5C). Similar results to the ones reported in this work have been found in several animal models involving neurological diseases, such as multiple sclerosis, Parkinson's disease, stroke and brain trauma [63].

Although the presence of progesterone receptors in microglia cells has not been well characterized, previous studies suggest that this hormone may decrease the local expression of cytokines and interleukins, reducing the expression of NOS and decreasing the release of reactive oxygen species (ROS). The main consequences of these factors would 
be the prevention of microglia cell migration and the transition to an activated cell phenotype [63].

\subsection{Progesterone effects on neuronal and inducible nitric oxide synthase}

Nitric oxide plays a critical role in visual processing and several retinal pathologies. NOS expression studies in mice retina suggest that nNOS is the predominant isoform of NOS and that immunoreactivity for nNOS reflects the majority of NO production in the normal retina [45]. In the present work, the results suggest that nNOS is altered in the far peripheral retina of the untreated rd10 mice. However, treatment with progesterone in control mice produces a significant decrease in nNOS in all areas of the retina (Fig. 6). Controversial results regarding the effect of gonadal hormones in nNOS expression have also been observed by other authors. Lima et al. demonstrated that estrogens stimulate the expression of nNOS in the preoptic area of female rats; this effect was not observed after progesterone administration [64]. Studies conducted in guinea pigs by Weiner et al. did not show a significant increase in nNOS and eNOS (endothelial nitric oxide synthase) after the administration of a dose of $5 \mathrm{mg} / \mathrm{kg}$ of progesterone [65]. Similarly, other studies showed that the administration of low concentrations of progesterone did not produce alterations in nNOS, while the administration of $17 \alpha$-estradiol or progesterone at high concentrations was able to inhibit its expression in a cerebellum homogenate [66].

Regarding iNOS, we have found a statistically significant decrease in the iNOS protein in the rd10 groups compared to the controls at P21. This decrease was not prevented with progesterone administration (Fig. 7). Other study demonstrated the presence of this protein in the photoreceptor external segments in the retina of rd2 mice (another RP animal model) before the peak of photoreceptor cell death [46]. To further examine whether the decrease in iNOS expression was related to the loss of photoreceptors in our model, we studied its expression at P13 (when the loss of photoreceptors is not evident) and at P18 (when there is a significant decrease in the number of rows of photoreceptors of rd10 mice). Our results show that there is no change in the rd10 retinal iNOS expression at P13 while there is a decrease at P18 when compared to control retinas (Fig. 8). This confirms our hypothesis that the decrease in the iNOS expression may be due to the changes and loss of photoreceptors that take place in the $\mathrm{rd} 10$ mice retina.

\subsection{Progesterone decrease lipid peroxidation}

Several authors propose a close relationship between inflammation and oxidative stress in various diseases, such as Parkinson's, diabetes, AMD or RP [67-70]. However, although both oxidative stress and inflammation occur because of a pathological situation, it is not clear whether oxidative stress is a cause of inflammation or a consequence of it.

Although progesterone does not have a typical antioxidant chemical structure, it reduces the damage caused by free radicals [71]. In addition, it has been observed that the administration of progesterone has beneficial effects, by reducing, in a dose-dependent manner, one of the cellular alterations derived from oxidative damage, such as lipid peroxidation, in different in vitro [72] and in vivo systems [73]. These results are in agreement with our suggestion that progesterone has an effect on the inhibition of retinal lipid peroxidation because it is able to reduce MDA concentrations in the rd10 mice to values similar to those found in control retinas (Fig. 9A).

In order to corroborate the in vivo effect observed in the progesterone induced lipid peroxidation inhibition, the effect of different concentrations of progesterone in an ex vivo oxidative damage model has been studied (Fig. 9B). To this end, the induction of lipid peroxidation in liver tissue has been carried out with iron salts and ascorbic acid.
This induction of peroxidation takes place due to the fact that the transition metals can also stimulate lipid peroxidation by catalysing the decomposition of the hydroperoxides formed ( $\mathrm{ROOH})$ to form alkoxy (RO-) and peroxy radicals (ROO-), which can also initiate chain reactions. In this sense, it has been demonstrated in the present work that progesterone also has the ability to inhibit ex vivo lipid peroxidation in a dose-dependent manner. It has been observed that there is a statistically significant and direct correlation between the dose of progesterone used and the concentration of MDA.

Our results corroborate a recent work that has reported the effect of Norgestrel, a progesterone analogue, on retinal microglia and macroglia in RP [74]. Furthermore, we have also explored progesterone effects on the expression of NOS and on lipid peroxidation. In this regard, it is important to highlight the significance of these results because peroxidation of lipid membranes can change membranes' functionality, permeability, metabolic processes and ionic balance, with important consequences in retinal physiology.

We may conclude that progesterone could be a good therapeutic option, not only in $\mathrm{RP}$, but also for other retinal diseases that have been associated with inflammation and lipid peroxidation.

\section{Declarations of interest}

None.

\section{Acknowledgements}

This work was supported by grants from Cardenal Herrera CEU University and Fundación Universitaria San Pablo CEU (FUSPBS-PPC 33/ 201 and Consolidación 2017/18).

\section{References}

[1] E.E. Baulieu, M. Schumacher, H. Koenig, I. Jung-Testas, Y. Akwa, Progesterone as neurosteroid: actions within the nervous system, Cell. Mol. Neurobiol. 16 (1996) 143-154.

[2] N.A. Compagnone, S.H. Mellon, Neurosteroids: biosynthesis and function of these novel neuromodulators, Front. Neuroendocrinol. 21 (2000) 1-56.

[3] E.E. Baulieu, P. Robel, M. Schumacher, Neurosteroids: beginning of the story, Int. Rev. Neurobiol. 46 (2001) 1-32.

[4] E. Plassart-Schiess, E.E. Baulieu, Neurosteroids: recent findings, Brain Res. Rev. 37 (2001) 133-140

[5] M. Schumacher, R. Hussain, N. Gago, J.P. Oudinet, C. Mattern, A.M. Ghoumari, Progesterone synthesis in the nervous system: implications for myelination and myelin repair, Front. Neurosci. 6 (2012) 10

[6] S.H. Mellon, L.D. Griffin, N.A. Compagnone, Biosynthesis and action of neurosteroids, Brain Res. Rev. 37 (2001) 3-12.

[7] S.L. Petersen, K.A. Intlekofer, P.J. Moura-Conlon, D.N. Brewer, J. Del Pino Sans, J.A. Lopez, Novel progesterone receptors: neural localization and possible functions, Front. Neurosci. 7 (2013) 164

[8] B. Lei, B. Mace, H.N. Dawson, D.S. Warner, D.T. Laskowitz, M.L. James, Anti-inflammatory effects of progesterone in lipopolysaccharide-stimulated BV-2 microglia, PLoS One9 (2014), e103969.

[9] P.D. Gupta, K.Sr. Johar, K. Nagpal, A.R. Vasavada, Sex hormone receptors in the human eye, Surv. Ophthalmol. 50 (2005) 274-284.

[10] P. Guarneri, R. Guarneri, C. Cascio, P. Pavasant, F. Piccoli, V. Papadopoulos, Neurosteroidogenesis in rat retinas, J. Neurochem. 63 (1994) 86-96.

[11] B.R. Rao, Isolation and characterization of an estrogen binding protein which may integrate the plethora of estrogenic actions in non-reproductive organs, J. Steroid Biochem. Mol. Biol. 65 (1998) 3-41.

[12] G.V. Callard, M. Drygas, D. Gelinas, Molecular and cellular physiology of aromatase in the brain and retina, J. Steroid Biochem. Mol. Biol. 44 (1993) 541-547.

[13] P. Guarneri, C. Cascio, D. Russo, S. D’Agostino, G. Drago, G. Galizzi, G. De Leo, F Piccoli, M. Guarneri, R. Guarneri, Neurosteroids in the retina: neurodegenerative and neuroprotective agents in retinal degeneration, Ann. N. Y. Acad. Sci. 1007 (2003) 117-128.

[14] C. Cascio, D. Russo, G. Drago, G. Galizzi, R. Passantino, R. Guarneri, P. Guarneri, 17beta-estradiol synthesis in the adult male rat retina, Exp. Eye Res. 85 (2007) 166-172.

[15] L.A. Wickham, J. Gao, I. Toda, E.M. Rocha, M. Ono, D.A. Sullivan, Identification of androgen, estrogen and progesterone receptor mRNAs in the eye, Acta Ophthalmol. Scand. 78 (2000) 146-153.

[16] M. Swiatek-De Lange, A. Stampfl, S.M. Hauck, H. Zischka, C.J. Gloeckner, C.A. Deeg, M. Ueffing, Membrane-initiated effects of progesterone on calcium dependent signaling and activation of VEGF gene expression in retinal glial cells, Glia 55 (2007) 1061-1073. 
[17] T. Nakazawa, H. Takahashi, M. Shimura, Estrogen has a neuroprotective effect on axotomized RGCs through ERK signal transduction pathway, Brain Res. 1093 (2006) 141-149.

[18] W.K. O'Steen, Ovarian steroid effects on light-induced retinal photoreceptor damage, Exp. Eye Res. 25 (1977) 361-369.

[19] I. Káldi, A. Berta, Progesterone administration fails to protect albino male rats against photostress-induced retinal degeneration, Eur. J. Ophthalmol. 14 (2004) 306-314.

[20] N. Lu, C. Li, Y. Cheng, A.L. Du, Protective effects of progesterone against high intraocular pressure-induced retinal ischemia-reperfusion in rats, Nan Fang Yi Ke Da Xue Xue Bao 28 (2008) 2026-2029.

[21] D. Feskanich, E. Cho, D.A. Schaumberg, G.A. Colditz, S.E. Hankinson, Menopausal and reproductive factors and risk of age-related macular degeneration, Arch. Ophthalmol. 126 (2008) 519-524.

[22] D.T. Hartong, E.L. Berson, T.P. Dryja, Retinitis pigmentosa, Lancet 368 (2006) 1795-1809.

[23] M. Chizzolini, A. Galan, E. Milan, A. Sebastiani, C. Costagliola, F. Parmeggiani, Good epidemiologic practice in retinitis pigmentosa: from phenotyping to biobanking, Curr. Genomics 12 (2011) 260-266.

[24] C. Hamel, Retinitis pigmentosa, Orphanet J. Rare Dis. 11 (2006) 40.

[25] G.J. Farrar, P.F. Kenna, P. Humphries, On the genetics of retinitis pigmentosa and on mutation-independent approaches to therapeutic intervention, EMBO J. 21 (2002) 857-864.

[26] Y. Mitamura, S. Mitamura-Aizawa, T. Nagasawa, T. Katome, H. Eguchi, T. Naito, Diagnostic imaging in patients with retinitis pigmentosa, J. Med. Invest. 59 (2012) $1-11$.

[27] S. Nakagawa, A. Oishi, K. Ogino, Y. Makiyama, M. Kurimoto, N. Yoshimura, Association of retinal vessel attenuation with visual function in eyes with retinitis pigmentosa, Clin. Ophthalmol. 8 (2014) 1487-1493.

[28] L.E. Smith, Bone marrow-derived stem cells preserve cone vision in retinitis pigmentosa, J. Clin. Invest. 114 (2004) 755-757.

[29] E.L. Berson, Retinitis pigmentosa and allied retinal diseases: electrophysiologic findings, Trans. Sect. Ophthalmol. Am. Acad. Ophthalmol. Otolaryngol. 81 (1976) OP659-666.

[30] J.N. Sahni, M. Angi, C. Irigoyen, F. Semeraro, M.R. Romano, F. Parmeggiani, Therapeutic challenges to retinitis pigmentosa: from neuroprotection to gene therapy, Curr. Genomics 12 (2011) 276-284.

[31] D.J. Spalton, A.H. Rahi, A.C. Bird, Immunological studies in retinitis pigmentosa associated with retinal vascular leakage, Br. J. Ophthalmol. 62 (1978) 183-187.

[32] N. Gupta, K.E. Brown, A.H. Milam, Activated microglia in human retinitis pigmentosa, late-onset retinal degeneration, and age-related macular degeneration, Exp. Eye Res. 76 (2003) 463-471.

[33] F. Doonan, T.G. Cotter, Norgestrel may be a potential therapy for retinal degenerations, Expert Opin. Investig. Drugs 21 (2012) 579-581.

[34] V. Sánchez-Vallejo, S. Benlloch-Navarro, R. López-Pedrajas, F.J. Romero, M. Miranda, Neuroprotective actions of progesterone in an in vivo model of retinitis pig mentosa, Pharmacol. Res. 99 (2015) 276-288.

[35] D.G. Stein, D.W. Wright, A.L. Kellermann, Does progesterone have neuroprotective properties?, Ann. Emerg. Med. 51 (2008) 164-172.

[36] D.G. Stein, Is progesterone a worthy candidate as a novel therapy for traumatic brain injury?, Dialogues Clin. Neurosci. 13 (2011) 352-359.

[37] D.T. Ramírez-Lamelas, S. Benlloch-Navarro, R. López-Pedrajas, R. Gimeno-Hernández, T. Olivar, D. Silvestre, M. Miranda, Lipoic acid and progesterone alone or in combination ameliorate retinal degeneration in an experimental model of hereditary retinal degeneration, Front. Pharmacol. 9 (2018) 469.

[38] L. Olivares-González, C. Martínez-Fernández de la Cámara, D. Hervás, J.M. Millán, R. Rodrigo, HIF-1 $\alpha$ stabilization reduces retinal degeneration in a mouse model of retinitis pigmentosa, FASEB J. (2018), fj201700985R.

[39] M.M. Bradford, A rapid and sensitive method for the quantitation of microgram quantities of protein utilizing the principle of protein-dye binding, Anal. Biochem. 72 (1976) 248-254.

[40] O.H. Lowry, N.J. Rosebrough, A.L. Farr, R.J. Randall, Protein measurement with the Folin phenol reagent, J. Biol. Chem. 193 (1951) 265-275.

[41] M.J. Richard, P. Guiraud, J. Meo, A. Favier, High-performance liquid chromatographic separation of malondialdehyde-thiobarbituric acid adduct in biological materials (plasma and human cells) using a commercially available reagent, J. Chromatogr. 577 (1992) 9-18.

[42] C.G. Fraga, A.L. Tappel, Damage to DNA concurrent with lipid peroxidation in rat liver slices, Biochem. J. 252 (1988) 893-896.

[43] A.I. Arroba, N. Alvarez-Lindo, N. van Rooijen, E.J. de la Rosa, Microglia-Müller glia crosstalk in the rd10 mouse model of retinitis pigmentosa, Adv. Exp. Med. Biol. (2014) 373-379.

[44] C. Jiang, K. Cui, J. Wang, Y. He, Microglia and cyclooxygenase-2: possible therapeutic targets of progesterone for stroke, Int. Immunopharmacol. 11 (2011) $1925-1931$.

[45] J. Blom, T. Giove, M. Deshpande, W.D. Eldred, Characterization of nitric oxide sig naling pathways in the mouse retina, J. Comp. Neurol. 520 (2012) 4204-4217.

[46] L.P. Yang, Y. Li, X.A. Zhu, M.O. Tso, Minocycline delayed photoreceptor death in rds mice through iNOS-dependent mechanism, Mol. Vis. 13 (2007) 1073-1082.

[47] S.P. Daiger, S.J. Bowne, L.S. Sullivan, Perspective on genes and mutations causing retinitis pigmentosa, Arch. Ophthalmol. 125 (2007) 151-158.

[48] C.K. Glass, K. Saijo, B. Winner, M.C. Marchetto, F.H. Gage, Mechanisms underlying inflammation in neurodegeneration, Cell 140 (2010) 918-934.
[49] E. Vecino, F.D. Rodriguez, N. Ruzafa, X. Pereiro, S.C. Sharma, Glia-neuron interactions in the mammalian retina, Prog. Retin. Eye Res. 51 (2016) 1-40.

[50] G. Chen, J. Shi, W. Jin, L. Wang, W. Xie, J. Sun, C. Hang, Progesterone administration modulates TLRs/NF-kappaB signaling pathway in rat brain after cortical contusion, Ann. Clin. Lab. Sci. 38 (2008) 65-74.

[51] C.L. Gibson, D. Constantin, M.J. Prior, P.M. Bath, S.P. Murphy, Progesterone suppresses the inflammatory response and nitric oxide synthase-2 expression following cerebral ischemia, Exp. Neurol. 193 (2005) 522-530.

[52] D.S. Pan, W.G. Liu, X.F. Yang, F. Cao, Inhibitory e8ffect of progesterone on inflammatory factors after experimental traumatic brain injury, Biomed. Environ. Sci. 20 (2007) 432-438.

[53] A.R. Sarkaki, M. Khaksari Haddad, Z. Soltani, N. Shahrokhi, M. Mahmoodi, Time and dose-dependent neuroprotective effects of sex steroid hormones on inflammatory cytokines after a traumatic brain injury, J. Neurotrauma 30 (2013) 47-54.

[54] G. Chen, J.X. Shi, M. Qi, H.X. Wang, C.H. Hang, Effects of progesterone on intestinal inflammatory response, mucosa structure alterations, and apoptosis following traumatic brain injury in male rats, J. Surg. Res. 147 (2008) 92-98.

[55] B. Chang, N.L. Hawes, M.T. Pardue, A.M. German, R.E. Hurd, M.T. Davisson, S. Nusinowitz, K. Rengarajan, A.P. Boyd, S.S. Sidney, M.J. Phillips, R.E. Stewart, R. Chaudhury, J.M. Nickerson, J.R. Heckenlively, J.H. Boatright, Two mouse retinal degenerations caused by missense mutations in the beta-subunit of rod cGMP phosphodiesterase gene, Vision Res. 47 (2007) 624-633.

[56] C. Gargini, E. Terzibasi, F. Mazzoni, E. Strettoi, Retinal organization in the retinal degeneration 10 (rd10) mutant mouse: a morphological and ERG study, J. Comp. Neurol. 500 (2007) 222-238.

[57] N. Cuenca, L. Fernández-Sánchez, L. Campello, V. Maneu, P. De la Villa, P. Lax, I. Pinilla, Cellular responses following retinal injuries and therapeutic approaches for neurodegenerative diseases, Prog. Retin. Eye Res. 43 (2014) 17-75.

[58] G. Luna, G.P. Lewis, C.D. Banna, O. Skalli, S.K. Fisher, Expression profiles of nestin and synemin in reactive astrocytes and Muller cells following retinal injury: a comparison with glial fibrillar acidic protein and vimentin, Mol. Vis. 16 (2010) 2511-2523.

[59] C. Espinosa-Garcia, I. Sayeed, S. Yousuf, F. Atif, E.G. Sergeeva, G.N. Neigh, D.G. Stein, Stress primes microglial polarization after global ischemia: Therapeutic potential of progesterone, Brain Behav. Immun. 66 (2017) 177-192.

[60] M.A. Arevalo, M. Santos-Galindo, E. Acaz-Fonseca, I. Azcoitia, L.M. Garcia-Segura, Gonadal hormones and the control of reactive gliosis, Horm. Behav. 63 (2013) 216-221.

[61] E.A. Sergeeva, C. Espinosa-Garcia, F. Atif, M.T. Pardue, D.G. Stein, Neurosteroid allopregnanolone reduces ipsilateral visual cortex potentiation following unilateral optic nerve injury, Exp. Neurol. 306 (2018) 138-148.

[62] M.H. Madeira, R. Boia, P.F. Santos, A.F. Ambrósio, A.R. Santiago, Contribution of microglia-mediated neuroinflammation to retinal degenerative diseases, Mediators Inflamm. 2015 (2015), 673090.

[63] S. Johann, C. Beyer, Neuroprotection by gonadal steroid hormones in acute brain damage requires cooperation with astroglia and microglia, J. Steroid Biochem. Mol. Biol. 137 (2013) 71-81.

[64] F.B. Lima, F.H. Ota, F.J. Cabral, B. Del Bianco Borges, C.R. Franci, Estrogen, but not progesterone, induces the activity of nitric oxide synthase within the medial preoptic area in female rats, Brain Res. 1578 (2014) 23-29.

[65] C.P. Weiner, I. Lizasoain, S.A. Baylis, R.G. Knowles, I.G. Charles, S. Moncada, Induction of calcium-dependent nitric oxide synthases by sex hormones, Proc. Natl. Acad. Sci. U. S. A. 91 (1994) 5212-5216.

[66] T. Hayashi, T. Ishikawa, K. Yamada, M. Kuzuya, M. Naito, H. Hidaka, A. Iguchi, Biphasic effect of estrogen on neuronal constitutive nitric oxide synthase via $\mathrm{Ca}(2+)$-calmodulin dependent mechanism, Biochem. Biophys. Res. Commun. 203 (1994) 1013-1019.

[67] R. Niranjan, The role of inflammatory and oxidative stress mechanisms in the pathogenesis of Parkinson's disease: focus on astrocytes, Mol. Neurobiol. 49 (2014) 28-38.

[68] K.N. Keane, V.F. Cruzat, R. Carlessi, P.I. de Bittencourt, Newsholme, molecular events linking oxidative stress and inflammation to insulin resistance and $\beta$-cell dysfunction, Oxid. Med. Cell Longev. 2015 (2015), 181643.

[69] S. Datta, M. Cano, K. Ebrahimi, L. Wang, J.T. Handa, The impact of oxidative stress and inflammation on RPE degeneration in non-neovascular AMD, Prog. Retin. Eye Res. 60 (2017) 201-218.

[70] C. Martínez-Fernández de la Cámara, M.D. Sequedo, U. Gómez-Pinedo, T. Jaijo, E. Aller, P. García-Tárraga, J.M. García-Verdugo, J.M. Millán, R. Rodrigo, Phosphodiesterase inhibition induces retinal degeneration, oxidative stress and inflammation in cone-enriched cultures of porcine retina, Exp. Eye Res. 111 (2013) 122-133.

[71] K.M. Webster, D.K. Wright, M. Sun, B.D. Semple, E. Ozturk, D.G. Stein, T.J. O'Brien, S.R. Shultz, Progesterone treatment reduces neuroinflammation, oxidative stress and brain damage and improves long-term outcomes in a rat model of repeated mild traumatic brain injury, J. Neuroinflammation 18 (12) (2015) 238.

[72] H. Vedder, N. Anthes, G. Stumm, C. Würz, C. Behl, J.C. Krieg, Estrogen hormones reduce lipid peroxidation in cells and tissues of the central nervous system, J. Neurochem. 72 (1999) 2531-2538.

[73] V.H. Ozacmak, H. Sayan, The effects of 17beta estradiol, 17alpha estradiol and progesterone on oxidative stress biomarkers in ovariectomized female rat brain subjected to global cerebral ischemia, Physiol. Res. 58 (2009) 909-912.

[74] S.L. Roche, A.M. Ruiz-Lopez, J.N. Moloney, A.M. Byrne, T.G. Cotter, Microglial-induced Müller cell gliosis is attenuated by progesterone in a mouse model of retinitis pigmentosa, Glia 66 (2018) 295-310. 